\title{
Synthesis of Single-crystalline $\mathrm{KNbO}_{3}$ Nanowires with a Sacrificial Template Method and Their Second Harmonic Generation Response
}

\author{
Cao Huiqun, You Cheng, Yu Jie, Cao Bo, Xin Hong, Yu Bin
}

Shenzhen University, Shenzhen 518060, China

\begin{abstract}
High yield orthorhombic single-crystalline $\mathrm{KNbO}_{3}$ nanowires with widths of $60 \sim 150 \mathrm{~nm}$ and lengths up to a few microns were synthesized, using $\mathrm{Nb}_{2} \mathrm{O}_{5}$ nanobelts as reactants as well as templates via the hydrothermal process. The products were determined by XRD, and the morphology and the structure were characterized by SEM, TEM, HRTEM, and SAED techniques. The growth direction of $\mathrm{KNbO}_{3}$ was determined to be the [001] crystallographic direction. Results show that synthesized nanowires exhibit a second harmonic generation (SHG) response, an efficient nanoscale second harmonic light source. The excellent nonlinear optical properties of $\mathrm{KNbO}_{3}$ have potential applications in nano-optical devices.
\end{abstract}

Key words: $\mathrm{KNbO}_{3}$; nanowires; hydrothermal reaction; $\mathrm{SHG}$ (second harmonic generation)

Template-directed synthesis represents a straightforward approach to generating one-dimensional (1D) nanostructures. In this approach, the template serves as a scaffold against which other materials are assembled with morphology complementary to that of the template. Both "hard" templates (such as carbon nanotubes or anodic aluminum oxide nanostructures) and "soft" templates (such as surfactant-directed micelles, reverse micelles, or block copolymers) have been developed ${ }^{[1]}$. The sacrificial template method has been proven to be a facile and efficient route for the synthesis of nanowires ${ }^{[2-4]}$. Few such templates have been identified and this approach has seldom been used to fabricate nanowires. The development of effective sacrificial templates for nanowire production could be of great significance.

$\mathrm{KNbO}_{3}$ is one of the most promising materials for nonlinear optical and electro-optical devices because of its large nonlinear susceptibility and high photorefractive coefficient ${ }^{[5,6]}$. One-dimensional nanostructures have novel characteristics, differing from bulk structures. They provide new opportunities for research and technological development. Wire like $\mathrm{KNbO}_{3}$ has been used to make a tunable nanometric light source which is compatible with physiological environments. It could be used to construct a novel form of subwavelength microscopy. The synthesis of $\mathrm{KNbO}_{3}$ nanowires is a topic of great interest ${ }^{[7,8]}$.

The hydrothermal method is widely used in the synthesis of nanowires. The synthesis of nanowires by this method can be directed by anisotropic crystal structures or using surfactant as a soft template. The hydrothermal method can be used to control morphology during the synthesis of $\mathrm{KNbO}_{3}{ }^{[9-19]}$. The effects of concentration, fill factor, reactive time, temperature, pressure, surfactant and structure directing agents in the hydrothermal synthesis of $\mathrm{KNbO}_{3}$ have been evaluated, but the synthesis of $\mathrm{KNbO}_{3}$ nanowires is still inadequate. There are problems with the structure and morphology that result in poor reproducibility and low yield. Huang and co-workers ${ }^{[9]}$ developed a

$\overline{\text { Received date: June 14, } 2015}$

Foundation item: National Natural Science Foundation of China (61178080); Shenzhen Science and Technology Planning (JCYJ 20140711144809545, JCYJ 20150324141711698, JCYJ 20150525092941022)

Corresponding author: Xin Hong, Ph. D., Associate Professor, College of Chemistry and Environmental Engineering, Shenzhen University, Shenzhen 518060, P. R. China, Tel: 0086-755-26557449, E-mail: xinghong70@126.com 
hydrothermal method to synthesize $\mathrm{KNbO}_{3}$ nanoneedles on a polycrystalline $\mathrm{Al}_{2} \mathrm{O}_{3}$ substrate using a 5 day reactive time. Wang et al. ${ }^{[12]}$ reported the formation of $\mathrm{KNbO}_{3}$ nanorods using hydrothermal synthesis and a $24 \mathrm{~h}$ reaction time. The sizes of the particles were more than $100 \mathrm{~nm}$, and rods were formed, not nanowires. Although much progress has been made in the synthesis of $\mathrm{KNbO}_{3}$ nanowires, a sacrificial template method to generate single-crystalline $\mathrm{KNbO}_{3}$ nanowires using a hydrothermal process has not been reported.

We describe a novel solution-phase template approach to synthesize single-crystalline $\mathrm{KNbO}_{3}$ nanowires. High yields of nanowires were obtained using $\mathrm{Nb}_{2} \mathrm{O}_{5}$ nanobelts as both template and reactant in a $\mathrm{KOH}$ based hydrothermal process. The evolution of the crystal shape and a possible mechanism of formation of $\mathrm{KNbO}_{3}$ nanowires were proposed. The nanowires were an efficient nanoscale second harmonic light source.

\section{Experiment}

The reactant $\mathrm{Nb}_{2} \mathrm{O}_{5}$ nanobelts used as a sacrificing template in the present study were synthesized as previously described ${ }^{[20]}$. In brief, $\mathrm{KNb}_{3} \mathrm{O}_{8}$ nanowires were produced using a molten-salt process. $1 \mathrm{~g} \mathrm{Nb}_{2} \mathrm{O}_{5}$ powder was mixed with $3.3 \mathrm{~g} \mathrm{KCl}$ and $3.9 \mathrm{~g} \mathrm{~K}_{2} \mathrm{SO}_{4}$ and ground for 20 30 min. The mixture was placed in a combustion boat, annealed at $800{ }^{\circ} \mathrm{C}$ for $4 \mathrm{~h}$ in a tube furnace, and naturally cooled to room temperature. The products were washed and dried. The $\mathrm{KNb}_{3} \mathrm{O}_{8}$ nanowires were added to a $2 \mathrm{~mol} / \mathrm{L}$ $\mathrm{HNO}_{3}$ solution and stirred for $36 \mathrm{~h}$. The products were filtered, washed with distilled water, and dried to form wire like $\mathrm{H}_{3} \mathrm{ONb}_{3} \mathrm{O}_{8}$. The $\mathrm{H}_{3} \mathrm{ONb}_{3} \mathrm{O}_{8}$ was heated at $650{ }^{\circ} \mathrm{C}$ for $1 \mathrm{~h}$ to form $\mathrm{Nb}_{2} \mathrm{O}_{5}$ nanobelts.

In a typical experimental procedure for the synthesis of $\mathrm{KNbO}_{3}$ nanowires, $0.26 \mathrm{~g} \mathrm{Nb}_{2} \mathrm{O}_{5}$ nanobelts, $25.5 \mathrm{~g} \mathrm{KOH}$ and $2.0 \mathrm{~g} \mathrm{KCl}$ were stirred in $30 \mathrm{~mL} \mathrm{H}_{2} \mathrm{O}$. The mixture was transferred into a $50 \mathrm{~mL}$ Teflon-lined autoclave, and the autoclave was kept in an oven at $150{ }^{\circ} \mathrm{C}$ for $3 \mathrm{~d}$. The composites were cooled to room temperature, washed with distilled water and ethanol, and dried at $100{ }^{\circ} \mathrm{C}$ for $12 \mathrm{~h}$. The samples were collected for characterization. The yield of $\mathrm{KNbO}_{3}$ nanowires was approximately $91 \%$, referred to reactant $\mathrm{Nb}_{2} \mathrm{O}_{5}$ nanobelts.

The morphology, the structure and the composition of the products were characterized by scanning electron microscopy (SEM, S-4177, Japan), transmission electron microscopy (TEM, JEM 2100HR, Japan), high resolution electron microscopy (HRTEM, JEM 2100HR, Japan), X-ray diffractometry (XRD, Rigaku D/max, Japan) with a $\mathrm{Cu} \mathrm{K \alpha}$ radiation source $(\lambda=0.15406 \mathrm{~nm})$, and selected area electron diffraction (SAED, JEM 2100HR Japan). A standard scanning confocal microscope (Leica TCS SP2) was used to characterize the nonlinear optical properties of the synthesized material.

\section{Results and Discussion}

\subsection{Morphology and structural properties}

Phase identification and crystalline product analysis were performed using powder X-ray diffraction (XRD). Fig.1 shows the XRD patterns of the intermediate products. All of the XRD peaks from Fig.1a can be assigned to the orthorhombic phase of $\mathrm{KNb}_{3} \mathrm{O}_{8}$ (JCPDS 75-2182) with lattice parameters of $a=0.8903 \mathrm{~nm}, b=2.116 \mathrm{~nm}$, and $c=0.3799 \mathrm{~nm}$. The crystal is a highly crystallized pure phase without any impurity. The phase of the intermediate product could be confirmed to be an orthorhombic $\mathrm{H}_{3} \mathrm{ONb}_{3} \mathrm{O}_{8}$ (JCPDS 44-0672) (Fig.1b) with lattice parameters of $a=0.91987 \mathrm{~nm}, b=2.24733 \mathrm{~nm}$, and $c=0.38237 \mathrm{~nm}$. The phase of the niobium oxide is monoclinic $\mathrm{Nb}_{2} \mathrm{O}_{5}$ (JCPDS 43-1042) with lattice parameters of $a=0.398 \quad 87$ $\mathrm{nm}, b=0.38506 \mathrm{~nm}$, and $c=1.27645 \mathrm{~nm}$. Fig.1d shows the XRD pattern of the final product. The diffraction peaks are in agreement with the Joint Committee on Power Diffraction Standard No. 32-0822, indicating that the product is highly crystallized pure phase $\mathrm{KNbO}_{3}$ without any impurities. The crystal structure of $\mathrm{KNbO}_{3}$ is orthorhombic phase with lattice parameters $a=0.56823 \mathrm{~nm}$ $b=0.57169 \mathrm{~nm}$, and $c=0.39882 \mathrm{~nm}$.

Fig.2a shows the SEM image of $\mathrm{KNb}_{3} \mathrm{O}_{8}$ product with diameters of 200 500 $\mathrm{nm}$ and the lengths ranged from several micrometers to tens of micrometers. The morphology of the wires does not change obviously after $\mathrm{HNO}_{3}$ treatment (Fig.2b). A large number of $\mathrm{Nb}_{2} \mathrm{O}_{5}$ nanobelts are observed after calcination. Their diameters are 300 700 nm (Fig.2c). Fig.2d shows the SEM image of the final product. A large number of $\mathrm{KNbO}_{3}$ nanowires are observed after the template-directed hydrothermal process. Their diameters are 50 150 $\mathrm{nm}$. The composition of single-crystal nanowires was calculated using the EDX spectrum (Fig.2e). The ratio of $\mathrm{K}: \mathrm{Nb}: \mathrm{O}$ is very close to $1: 1: 3$, which is consistent with the stoichiometry of $\mathrm{KNbO}_{3}$.

TEM analysis provides an insight into the structural features of the $\mathrm{KNbO}_{3}$ nanowires. Fig.3a shows the TEM

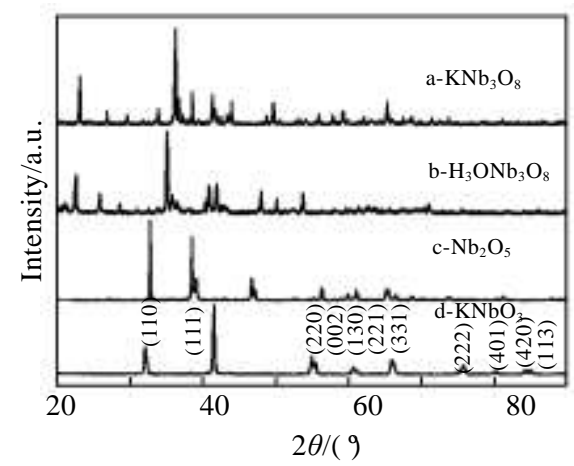

Fig.1 XRD patterns of the intermediate products 


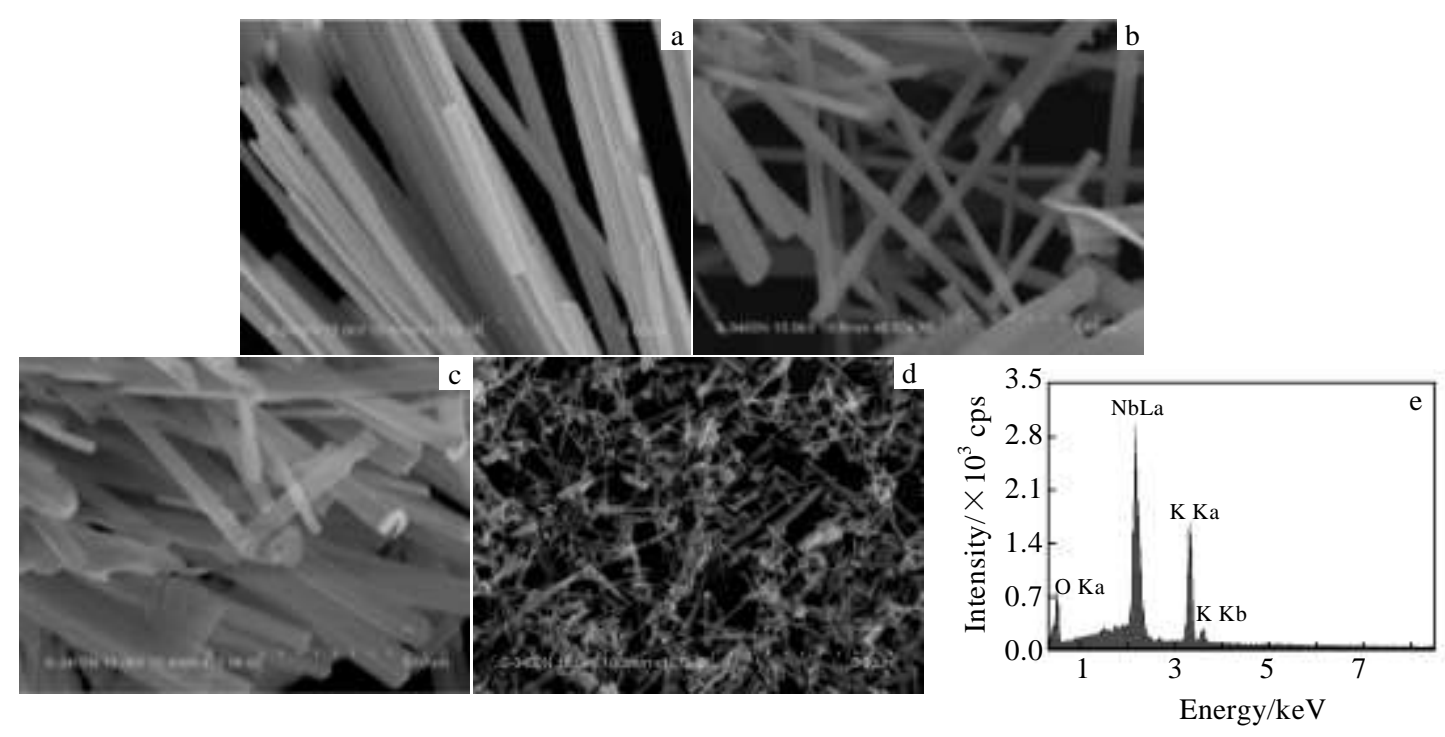

Fig.2 SEM images of synthesized $\mathrm{KNb}_{3} \mathrm{O}_{8}(\mathrm{a}), \mathrm{H}_{3} \mathrm{ONb}_{3} \mathrm{O}_{8}$ (b), $\mathrm{Nb}_{2} \mathrm{O}_{5}$ (c), and $\mathrm{KNbO}_{3}$ (d); EDX spectrum for Fig.2d (e)

image of an individual $\mathrm{KNbO}_{3}$ nanowire. The diameter is 80 $\mathrm{nm}$. The surfaces of the nanowires are smooth and regular. The HRTEM image demonstrates a well-defined lattice fringe on the nanowire. As shown in Fig.3b, the lattice fringe spacings along the growth and lateral directions are 0.397 and $0.404 \mathrm{~nm}$, respectively. This is indexed as (001) and (110) planes of an orthorhombic $\mathrm{KNbO}_{3}$ crystal. This is in agreement with the selected area electron diffraction (SAED) results shown in Fig.3c. The sharp diffraction spots in the SAED pattern are consistent with single-crystalline nanowires that grow in the [001] crystallographic direction.

\subsection{Proposed formation mechanism}

The structural evolution of $\mathrm{KNbO}_{3}$ was descried as the following reactions.

$$
\begin{aligned}
& 6 \mathrm{Nb}_{2} \mathrm{O}_{5}+2 \mathrm{KCl}+\mathrm{K}_{2} \mathrm{SO}_{4} \rightarrow 4 \mathrm{KNb}_{3} \mathrm{O}_{8}+\mathrm{Cl}_{2}+\mathrm{SO}_{2} \\
& \mathrm{KNb}_{3} \mathrm{O}_{8} \rightarrow \mathrm{H}_{3} \mathrm{ONb}_{3} \mathrm{O}_{8} \\
& 2 \mathrm{H}_{3} \mathrm{ONb}_{3} \mathrm{O}_{8} \rightarrow 3 \mathrm{Nb}_{2} \mathrm{O}_{5}+\mathrm{H}_{2} \mathrm{O} \\
& \mathrm{Nb}_{2} \mathrm{O}_{5}+\mathrm{KOH} \rightarrow \mathrm{KNbO}_{3}
\end{aligned}
$$

The synthesis of $\mathrm{KNbO}_{3}$ occurs in a $\mathrm{KOH}$ solution via dissolution precipitation, described as the following three reactions:

$$
\begin{aligned}
& 3 \mathrm{Nb}_{2} \mathrm{O}_{5}+8 \mathrm{OH}^{-} \rightarrow \mathrm{Nb}_{6} \mathrm{O}_{19}{ }^{8-}+4 \mathrm{H}_{2} \mathrm{O} \\
& \mathrm{Nb}_{6} \mathrm{O}_{19}{ }^{8-}+34 \mathrm{OH}^{-} \rightarrow 6 \mathrm{NbO}_{6}{ }^{7-}+17 \mathrm{H}_{2} \mathrm{O} \\
& \mathrm{NbO}_{6}{ }^{7-}+\mathrm{K}^{+}+3 \mathrm{H}_{2} \mathrm{O} \rightarrow \mathrm{KNbO}_{3}+6 \mathrm{OH}^{-}
\end{aligned}
$$

The hydrothermal synthesis has no catalyst serving as the energetically favorable site for the absorption of reactant molecules and no template to guide the directional growth of nanowires. The driving force for the anisotropic growth is mainly derived from the inherent crystal structure of the materials. In our process, the $\mathrm{Nb}_{2} \mathrm{O}_{5}$ nanobelts are converted to soluble $\mathrm{Nb}_{6} \mathrm{O}_{19}{ }^{8-}$. A minimal reorganization of the parent solid structure is the key to the formation of the single- crystalline $\mathrm{KNbO}_{3}$ nanowires. The atomic arrangement in the template is unaffected during the course of this reaction, so $\mathrm{KNbO}_{3}$ inherits the shape of the $\mathrm{Nb}_{2} \mathrm{O}_{5}$ precursor. The formed particles become anisotropic. These anisotropic clusters serve as seeds for subsequent growth along the smallest surface energy direction, resulting
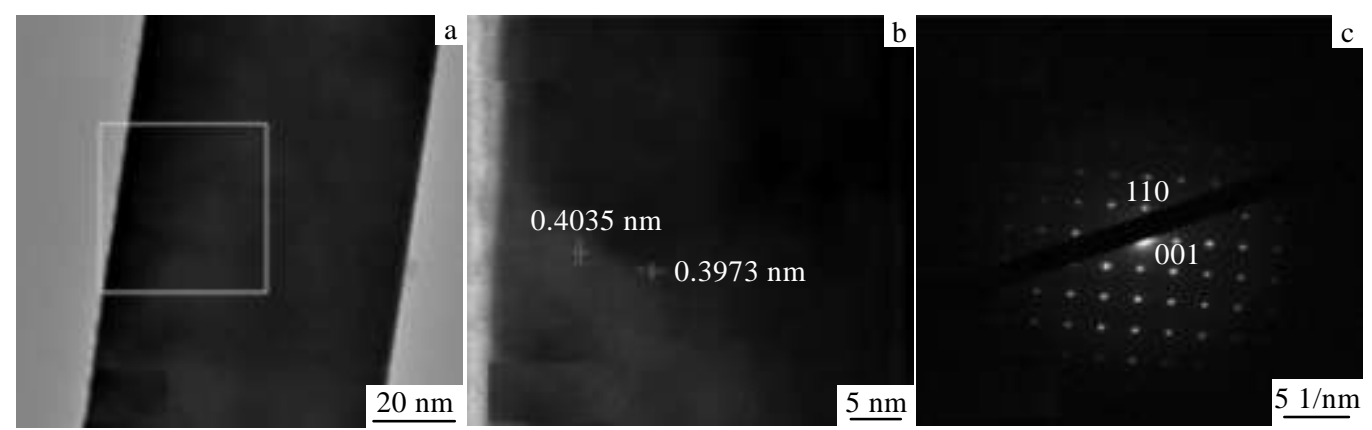

Fig.3 TEM image of the $\mathrm{KNbO}_{3}$ nanowire (a), HRTEM image from the side of the nanowire (white square in Fig3.a) (b) and SAED pattern from the same area of the nanowire (c) 
in the formation of nanowires ${ }^{[21]}$. Our experiments prove the viewpoint. Using a reaction time of $12 \mathrm{~h}$, a large number of cluster $\mathrm{KNbO}_{3}$ nanowires are formed (Fig.4a). The nanowires are a highly crystallized pure phase without any impurity identified by XRD. The length of the nanowires is short. With the extending of reactive time, the cluster gradually disappear, as shown in Fig.4b. Longer nanowires are formed when the reaction time is prolonged.

At the same time, there is a strong dependence of $\mathrm{KNbO}_{3}$ shape and reaction rate on composition of the starting mixture. $\mathrm{KOH}$ is essential for wire-shaped growth. Without $\mathrm{KOH}$ in the reaction system, the particles are isotropic and grow in a radial direction. Nanowires are not formed. Reaction (5) is fast and reaction (6) is very slow at $150{ }^{\circ} \mathrm{C}$. The higher the $\mathrm{KOH}$ concentration, the larger the solubility of $\mathrm{Nb}_{2} \mathrm{O}_{5}$, which benefit the formation of $\mathrm{KNbO}_{3}$. However, if the $\mathrm{KOH}$ concentration is too high, reaction (7) is inhibited. The proper concentration of $\mathrm{KOH}$ is needed. The reaction consumes $\mathrm{KOH}$ and produces $\mathrm{H}_{2} \mathrm{O}$. This lowering of $\mathrm{KOH}$ concentration reduces the reaction rate, increasing the time required to form $\mathrm{KNbO}_{3}$ nanowires. A small amount of $\mathrm{KCl}$ introduced into the solution increases the $\mathrm{K}^{+}$ concentration, which increases the formation of $\mathrm{KNbO}_{3}$. The $\mathrm{KNbO}_{3}$ nanowires are synthesized with a high yield in short time. The exact transformation mechanism from $\mathrm{Nb}_{2} \mathrm{O}_{5}$ nanobelts to $\mathrm{KNbO}_{3}$ single-crystalline nanowires is not completely understood. Further investigation of the detailed reaction process is underway.

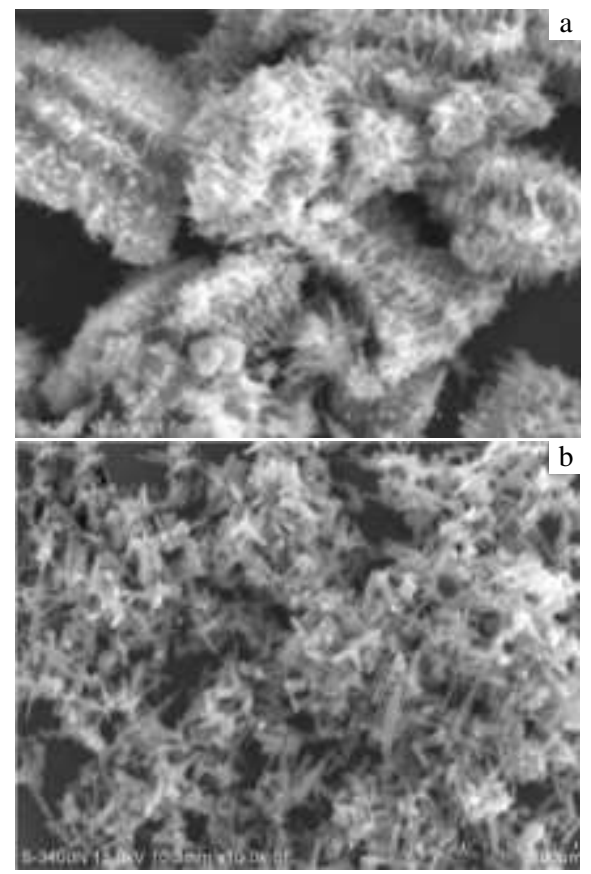

Fig.4 SEM images of synthesized $\mathrm{KNbO}_{3}$ at $12 \mathrm{~h}$ (a) and $24 \mathrm{~h}(\mathrm{~b})$

\subsection{Nonlinear optical properties}

After producing $\mathrm{KNbO}_{3}$ nanowires, we decided to examine their second-harmonic generation response, a second-order nonlinear optical phenomenon. We first used a standard scanning confocal microscope (Leica TCS SP2) to test whether our synthesized nanowires generated any SHG signals. This approach permits a fast characterization of many nanowires. Unlike fluorescence, the SHG process occurs without nonradiative energy loss and involves only virtual electron energy transition. Therefore, SHG structures do not bleach over time and emit a stable, nonblinking signal that does not saturate with increasing of excitation power. Prior to imaging, the $\mathrm{KNbO}_{3}$ precipitate was diluted with deionized water, deposited on a glass slide, and examined with a confocal microscope. The laser beam from a Ti: sapphire oscillator, operated at a $860 \mathrm{~nm}$ wavelength with $150 \mathrm{fs}$ pulse and $80 \mathrm{MHz}$ repetition rate, was focused to a $20 \mu \mathrm{m}$ diameter on the sample. The average power was $250 \mathrm{~mW}$. The SHG signal at $430 \mathrm{~nm}$ was then collected with an oil immersion microscope objective and recorded on a photomultiplier that filtered out the pump wavelength. The polarization of the pump beam was rotated with a half-wave plate to obtain a polarizationdependent SHG response. During the measurement, the incident laser polarization was varied by $10^{\circ}$, and at each angle the SHG signal was recorded by acquiring an image (Fig.5 inset). All images were analyzed using a program which detected the highest intensity spot on each image and extrapolated the intensity. A polar plot of the SHG maximum value for each incident polarization angle was created by plotting the maximum normalized intensity values versus the polarization angle. Fig.5 shows the polarization- dependent response of the SHG emission for a single $\mathrm{KNbO}_{3}$ nanowire.

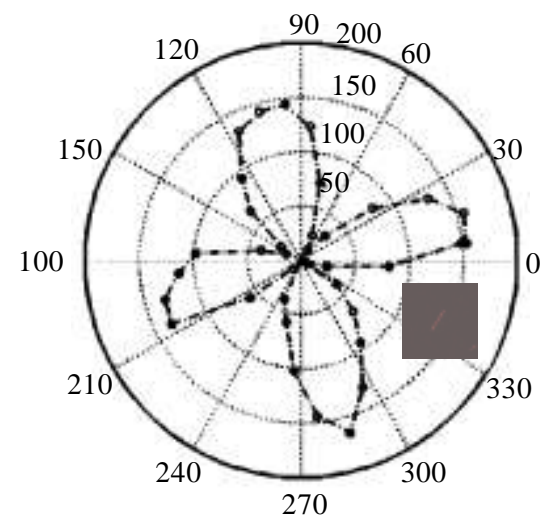

Fig.5 Polarization response of the SHG from a single $\mathrm{KNbO}_{3}$ nanowire in polar coordinates (inset: confocal image of the $\mathrm{SHG}$ signal from a single $\mathrm{KNbO}_{3}$ nanowire) 


\section{Conclusions}

1) High yield single-crystalline $\mathrm{KNbO}_{3}$ nanowires can be synthesized using a sacrificial template process. The process does not require an organic additive.

2) Growth of the crystal occurs in the [001] crystallographic direction. The $\mathrm{Nb}_{2} \mathrm{O}_{5}$ nanobelt template plays an important role in the formation of $\mathrm{KNbO}_{3}$ nanowires. Different morphology nanowires can be obtained by altering the reaction time. Our study provides a new method for the direct growth of single-crystalline nanowires and related materials.

3) The nanowires have a strong SHG response, which means they are an efficient nanoscale second harmonic light source.

\section{References}

1 Gates B, Wu Y Y, Yin Y D et al. Journal of the American Chemical Society[J], 2001, 123: 11500

$2 \mathrm{Wu} \mathrm{C} \mathrm{Y}, \mathrm{Yu} \mathrm{S} \mathrm{H}$, Chen S F et al. Journal of Materials Chemistry[J], 2006, 16: 3326

3 Li Z Q, Yang H, Ding Y et al. Dalton Transactions[J], 2006, 1: 149

4 Yuan X S, Yang B J, Hao J W et al. Crystal Research and Technology[J], 2011, 46: 1296

5 Grange R, Choi J W, Hsieh C L. Applied Physics Letters[J], 2009, 95: 143105

6 Dutto F, Raillon C, Schenk K et al. Nano Letters[J], 2011, 11: 2517

7 Nakayama Y, Pauzauskie P J, Radenovic A et al. Nature[J],
2007, 447: 1098

8 Pauzauskie P J, Radenovic A, Trepagnier E et al. Nature Materials $[\mathrm{J}], 2006,5: 97$

9 Wang Y, Chen Z, Ye Z Z et al. Journal of Crystal Growth[J], 2012, 341: 42

10 Magrez A, Vasco E, Seo J W et al. Journal of Physical Chemistry $B[\mathrm{~J}], 2006,110$ : 58

11 Ding Q P, Yuan Y P, Xiong X et al. The Journal of Physical Chemistry $C[\mathrm{~J}], 2008,112: 18846$

12 Wang G Z, Selbach S M, Yu Y D et al. Cryst Eng Comm[J], 2009, 11: 1958

13 Liu J W, Chen G, Li Z H et al. International Journal of Hydrogen Energy [J], 2007, 32: 2269

14 Wang Y, Yi Z G, Li Y X et al. Ceramics International[J], 2007, 33: 1611

15 Santos I C M S, Loureiro L H, Silva M F P et al. Polyhedron[J], 2002, 21: 2009

16 Wang C, Hou Y D, Ge H Y et al. Rare Metal Materials and Engineering[J], 2010, 39(2): 361 (in Chinese)

17 Cao H Q, Wang F, Yu B et al. Rare Metal Materials and Engineering[J], 2010, 39(2): 487 (in Chinese)

18 Li B, Hakuta Y, Hayashi H et al. Journal of Supercritical fluids[J], 2005, 35: 254

19 Liu J F, Li X L, Li Y D. Journal of Crystal Growth[J], 2003, 247: 419

20 Li L, Deng J, Chen J et al. Chemistry of Materials[J], 2009, 21: 1207

21 Shi L, Xu Y M, Li Q. The Journal of Chemical Physics C[J], 2009, 113: 1795

\title{
牺牲模板法制备铌酸钾纳米棒单晶及其二次谐波产生
}

\author{
曹慧群, 游 诚, 于 杰, 曹 博, 辛 红, 于 斌 \\ (深圳大学, 广东 深圳 518060)
}

\begin{abstract}
摘 要: 首次使用五氧化二铌纳米棒作为原料通过水热反应合成出 $60 \sim 150 \mathrm{~nm}$ 宽、几个微米长的高产率、斜方晶系铌酸钾纳米棒晶体。 使用 $\mathrm{X}$ 射线衍射仪 (XRD)、扫描电镜 (SEM)、透射电镜(TEM)、高分辨透射电镜(HRTEM)、选区电子衍射技术(SAED)对铌酸钾纳米棒 晶体的形貌和结构进行了表征。合成的铌酸钾纳米棒表现出二次谐波产生响应, 并发射出高效率的纳米二次谐波光线。铌酸钾纳米棒 晶体沿着[001]方向生长。合成的银酸钾纳米棒以其优异的非线性光学性能在纳米光学器件中的应用具有很好的发展前景。
\end{abstract}

关键词：铌酸钾；纳米线; 水热法; 二次谐波产生

作者简介: 曹慧群, 女, 1976 年生, 博士, 副教授, 深圳大学化学与环境工程学院, 广东 深圳 518060, 电话: 0755-26557449, E-mail: chq0524@163.com 